\title{
Macrophage migration inhibitory factor antagonist (S,R)3-(4-hydroxyphenyl)-4,5-dihydro-5-isoxazole acetic acid methyl ester attenuates inflammation and lung injury in rats with acute pancreatitis in pregnancy
}

\author{
YU ZHOU ${ }^{1-3}$, LIANG ZHAO ${ }^{1-3}$, FANGCHAO MEI ${ }^{1-3}$, YUPU HONG $^{1-3}$, HE XIA $^{1-3}$, \\ TENG ZUO $^{1}$, YOUMING DING ${ }^{1}$ and WEIXING WANG ${ }^{1}$ \\ ${ }^{1}$ Department of General Surgery; ${ }^{2}$ Central Laboratory; ${ }^{3}$ Hubei Key Laboratory of Digestive System Disease, \\ Renmin Hospital of Wuhan University, Wuhan, Hubei 430060, P.R. China
}

Received November 10, 2017; Accepted February 14, 2018

DOI: $10.3892 / \mathrm{mmr} .2018 .8672$

\begin{abstract}
Macrophage migration inhibitory factor (MIF) is an inflammatory cytokine involved in many acute and chronic inflammatory diseases. However, its role in acute lung injury associated with acute pancreatitis in pregnancy (APIP) has not yet been elucidated. The present study was undertaken to clarify the effect and potential mechanism of MIF antagonist (S,R)3-(4-hydroxyphenyl)-4,5-dihydro-5-isoxazole acetic acid methyl ester (ISO-1) in the development of acute lung injury in rats with APIP. Eighteen late-gestation SD rats were randomly assigned to three groups: Sham operation (SO) group, APIP group, and ISO-1 group. All the rats were sacrificed $6 \mathrm{~h}$ after modeling. The severity of pancreatitis was evaluated by serum amylase (AMY), lipase (LIPA), tumor necrosis factor (TNF)- $\alpha$, interleukin (IL)-1 $\beta$ and IL- 6 and assessing the histopathological score. Lung injury was determined by performing histology and inflammatory cell infiltration investigations. Western blot analysis was used to detect the protein expression of MIF, phosphorylated and total P38 and nuclear factor- $\mathrm{KB}$ $(\mathrm{NF}-\mathrm{\kappa B})$ protein in lungs. The results showed that MIF was upregulated in the lung of APIP rats. Compared with APIP group, the intervention of ISO-1 alleviated the pathological injury of the pancreas and lungs, decreased serum AMY and
\end{abstract}

Correspondence to: Professor Weixing Wang, Department of General Surgery, Renmin Hospital of Wuhan University, 238 Jiefang Road, Wuhan, Hubei 430060, P.R. China

E-mail: sate.1lite@163.com

Abbreviations: MIF, macrophage migration inhibitory factor; APIP, acute pancreatitis in pregnancy; ISO-1, (S,R)3-(4-hydroxyphenyl)-4,5-dihydro-5-isoxazole acetic acid methyl ester; TNF- $\alpha$, tumor necrosis factor- $\alpha$; IL, interleukin; MPO, myeloperoxidase; NF- $\mathrm{B}$, nuclear factor- $\kappa \mathrm{B}$

Key words: macrophage migration inhibitory factor, lung injury, acute pancreatitis, pregnancy, inflammatory response
LIPA, attenuated serum concentrations of TNF- $\alpha$, IL-1 $\beta$, and IL-6, reduced the number of MPO-positive cells in the lung and inhibited the activation of P38MAPK and NF- $\kappa$ B. These results suggest that MIF is activated in lung injury induced by APIP. Furhtermore, the present findings indicate that the MIF antagonist ISO-1 has a protective effect on lung injury and inflammation, which may be associated with deactivating the P38MAPK and NF- $\mathrm{KB}$ signaling pathway.

\section{Introduction}

Acute pancreatitis in pregnancy (APIP) is a rare event, attacking approximately $1 / 10,000$ to $1 / 1,000$ pregnancies $(1,2)$, thereby the information on maternal and fetal complications is limited. Although less frequent in clinical practice, it was associated with up to $5 \%$ of maternal deaths and fetal loss (3). APIP usually occurs in the third trimester of pregnancy (4), and gallstones are the most common cause and responsible for more than $60 \%$ of cases $(1,2)$. As in any other disease associated with pregnancy, APIP is associated with greater concerns as it deals with two lives.

It has been widely accepted that the activation of trypsinogen leads to self-digestion of pancreatic acinar cells and then results in acute pancreatitis. Acute pancreatitis is frequently complicated by an intensive systemic inflammatory response, in which increased infiltration of inflammatory cells is observed in multiple organs, such as the liver, kidney, and lung, further leading to multiple organ dysfunction syndrome (5). Among these organs, the lung is the most vulnerable one (6). Acute lung injury is reported to occur in $10-25 \%$ of acute pancreatitis cases, and it is responsible for up to $60 \%$ of acute pancreatitis-associated deaths (7). Additionally, accumulated studies have demonstrated that acute pancreatitis-triggered systemic inflammatory response causes acute lung injury (8). Moreover, in acute pancreatitis-induced lung injury, inflammatory cascade involving the activation and release of various inflammatory cytokines, such as nuclear factor- $\kappa \mathrm{B}$ $(\mathrm{NF}-\kappa \mathrm{B})$, tumor necrosis factor (TNF)- $\alpha$, interleukin (IL)-1 $\beta$ and IL-6, was significantly induced (9). Therefore, reducing 
proinflammatory mediators might be a good therapeutic strategy to attenuate acute lung injury associated with APIP.

Macrophage migration inhibitory factor (MIF) is a structurally unique pleiotropic cytokine that plays an important role as an upstream regulator of innate and acquired immunity as well as in cellular redox signaling (10). It regulates inflammatory response through extra- and intracellular processes, such as binding to a receptor complex made of CD74 with or without CD44, CXCR2, and CXCR4 to initiate intracellular signaling $(11,12)$. Through these interactions, MIF negatively or positively regulates MAPKs (13). For example, MIF induced the phosphorylation of P38MAPK (14), which may ascribe to reduce the expression of MKP-1, a critical phosphatase in physiological counter-regulatory MIF-glucocorticoids (GCs) dyad (15). In most cases, MIF is recognized as a pro-inflammatory cytokine whose neutralizing antibody (16) or small-molecule inhibitor (17) is used for suppressing inflammation with high levels of MIF in blood circulation or local tissue in various animal models, such as severe sepsis (18), rheumatoid arthritis (19), allergic airway inflammation (20), colitis (21) and chronic obstructive pulmonary disease (22). Therefore, a promising therapeutic approach to diminish pathological inflammation is to inhibit the production and/or biological activity of MIF.

(S,R)3-(4-hydroxyphenyl)-4,5-dihydro-5-isoxazole acetic acid methyl ester (ISO-1), a small molecule antagonist of MIF, is from the isoxazole series, whose active compounds were identified by virtue of their ability to inhibit the tautomerase activity of MIF (17). ISO-1 inhibits Toll-like receptor-4 (TLR-4)-induced proinflammatory cytokine production from monocytes (23), the macrophage release of TNF- $\alpha$ from lipopolysaccharide (LPS)-stimulated mice and is moderately protective in a clinically relevant model of sepsis when administered intraperitoneally (17).

However, whether MIF inhibition by ISO-1 is effective in protecting against acute lung injury induced by APIP has not yet been elucidated. We hypothesized that MIF is involved in the pathogenesis of acute lung injury induced by APIP, and MIF antagonist ISO-1 can protect against the lung injury. Therefore, in the present study, we attempted to investigate the effect and potential mechanism of MIF antagonist ISO-1 in the development of acute lung injury in rats with APIP. The results may provide a theoretical basis for the treatment of acute lung injury associated with APIP.

\section{Materials and methods}

Antibodies and reagents. ISO-1 and sodium taurocholate were obtained from Sigma-Aldrich (Merck KGaA, Darmstadt, Germany). The primary antibodies against P38, phosphorylated-P38, NF- $\kappa \mathrm{B} / \mathrm{p} 65$ and TNF- $\alpha$ were purchased from Cell Signaling Technology Inc. (Danvers, MA, USA). MIF primary antibody was from Abcam (Cambridge, CA). Rat anti-MPO antibody was from Wuhan Goodbio Technology Co., Ltd. (Wuhan, China). Rat TNF- $\alpha$, IL-1 $\beta$, IL-6 enzyme-linked immunosorbent assay (ELISA) kits were purchased from Cusabio Corp (Wuhan, China).

Animals. Eighteen pregnant Sprague-Dawley (SD) rats (17-18 days of the first gestation, weighing 390-450 g) were obtained from the Experimental Animals Center of Huazhong University of Science and Technology (Wuhan, China). The animals were kept under standardized conditions with an ambient temperature of $23 \pm 2^{\circ} \mathrm{C}$ and a $12 \mathrm{~h}$ light and dark cycle. Before the induction of pancreatitis, the animals were fed standard laboratory rodent chow, allowed free access to sterile water. All rats were fasted for $12 \mathrm{~h}$ prior to the modeling while given water ad libitum. All animal experiments in this study were reviewed and approved by the Ethics Committee of Wuhan University and performed in compliance with the ARRIVE guidelines.

Experimental model and groups. Rats we re anesthetized with isoflurane (induced with 5\% isoflurane and maintaining with $3 \%$ in $21 /$ min oxygen flow in a sealed container) and underwent standardized surgical procedures as described previously (24) and minor steps were revised. Briefly, the APIP rat model was induced by retrograde infusion of $5 \%$ sodium taurocholate solution $(1 \mathrm{ml} / \mathrm{kg})$ into the biliary-pancreatic duct at a constant speed of $0.10 \mathrm{ml} / \mathrm{min}$. The pancreas appeared to be hemorrhaged and necrotic after 5 mins, indicating the APIP model was induced successfully. After closure, $20 \mathrm{ml} / \mathrm{kg}$ body weight of saline solution was compensated back subcutaneously for fluid loss.

The rats were randomly assigned into three experimental groups: i) Sham operation group (SO group); ii) APIP group; and iii) ISO-1 + APIP group (ISO-1 group), including 6 rats in each group. All SO group underwent the same procedures but were retrogradely infused with equivalent saline water instead. The rats of the ISO-1 group were intraperitoneally administered with $3.5 \mathrm{mg} / \mathrm{kg}$ ISO-1 (dissolved in 5\% DMSO diluted in saline) $30 \mathrm{~min}$ before the modeling. The dosage and time for ISO-1 were based on our previous study (25), which was non-toxic and effective. The rats in SO and APIP groups received an equivalent volume of vehicle (5\% DMSO diluted in saline) instead of ISO-1 before the operation.

Collection of blood and tissue samples. All the rats were sacrificed at $6 \mathrm{~h}$ after modeling, which was based on our earlier study (2). Blood samples were collected by inferior vena cava puncture and the serum was stored at $-80^{\circ} \mathrm{C}$ for further analysis. Subsequently, pancreas and lung tissues were excised and fixed in $4 \%$ polyoxymethylene for histological detection or were frozen immediately in liquid nitrogen and stored at $-80^{\circ} \mathrm{C}$ for the following assay.

Serum enzyme activity assay. Serum amylase (AMY) and lipase (LIPA) levels were measured by a full automatic biochemical analyzer (Olympus AU680; Olympus, Tokyo, Japan) using standard techniques.

Histopathology analysis. The pancreatic and lung specimens were fixed in $4 \%$ polyformaldehyde, embedded with paraffin, sectioned at $4 \mu \mathrm{m}$ thick, and sequentially stained with hematoxylin and eosin (H\&E). All slides were assessed under the optical microscope (Olympus Optical Ltd., Tokyo, Japan) by 3 experienced pathologists who are blind to the research. The Pancreatic histological assessment was determined by edema, hemorrhage, vacuolization, inflammatory cell infiltration, and acinar necrosis according to the standard scale system 
described by Schmidt et al (26). Similarly, lung injury was assessed using a scale for interalveolar septal thickening, alveolar hemorrhage and inflammatory cell infiltration and fibrosis, as described by Werner et al (27).

ELISA. The serum concentrations of TNF- $\alpha$, IL-1 $\beta$, and IL-6 were detected by enzyme-linked immunosorbent assay (ELISA) using corresponding ELISA kits according to the manufacturer's protocols. The absorbance was read using an automated microplate reader at $450 \mathrm{~nm}$ and the concentrations were calculated according to the standard curve run on each assay plate. All samples were duplicated 3 times.

Immunofluorescence assay. Myeloperoxidase (MPO), the marker of neutrophil infiltration, was detected in the lung by immunofluorescence analyses. Briefly, following xylene deparaffinization and hydration using a graded series of ethanol solutions, the slides were boiled for $10 \mathrm{~min}$ at $121^{\circ} \mathrm{C}$ in a pressure cooker containing $10 \mathrm{mM}$ citrate buffer ( $\mathrm{pH}$ 9.0) for epitope retrieval. Subsequently, the slides were cooled to room temperature and rinsed in phosphate-buffered saline (PBS). After permeabilization with $0.2 \%$ Triton X-100 for $45 \mathrm{~min}$, the slides were washed with PBS and then blocked with $10 \%$ normal donkey serum to eliminate the nonspecific fluorescence. The sections were incubated with the primary antibody against MPO (1:200) at $4^{\circ} \mathrm{C}$ overnight in a humidity box. And followed by the fluorescence-labeled secondary antibodies at room temperature for $1 \mathrm{~h}$. Nuclei were counter-stained with DAPI. The negative control experiments were performed in which PBS was substituted for the primary antibody. All sections were examined and photographed using an automatic fluorescence microscope (Olympus Optical Ltd.) under blind conditions. And the staining was analyzed by Image Pro-Plus 6.0 system (Media Cybernetics Inc., Rockville, MD, USA).

Western blot analysis. The expression of MIF, phosphorylated-P38, P38, TNF- $\alpha$ and NF- $\mathrm{KB}$ in the lung were determined by western blot analysis. Lung tissues were homogenized and lysed on ice with lysis buffer (nuclear-cytosol extraction kit; Applygen Technologies Inc., Beijing, China) in the presence of protease and phosphorylase inhibitor cocktail (Roche Diagnostics, Mannheim, Germany). Lysates were collected, and the concentrations of protein were detected with BCA protein assay. In brief, equal amounts of protein samples were electrophoresed on 10 or $12 \%$ sodium dodecyl sulfate-polyacrylamide gels (SDS-PAGE) and then transferred to polyvinylidene difluoride (PVDF) membranes (Millipore). After blocking with 5\% fat-free milk dissolving in Tris-buffered saline containing $0.1 \%$ Tween-20 (TBST) at room temperature for $2 \mathrm{~h}$, the membranes were subsequently incubated with the primary antibodies (all of them were diluted as recommended 1:1,000) overnight at $4^{\circ} \mathrm{C}$. Following washing with TBST ( 5 min $x 3$ ), the membranes were incubated with fluorescently-labeled secondary antibody at room temperature for 1-2 $\mathrm{h}$. Then the specific protein bands were scanned by Odyssey Infrared Imaging System (LI-COR Biosciences, Lincoln, NE, USA) according to the manufacturer's instructions. The relative band intensity was quantified by Quantity One 4.6.2 software (Bio-Rad Laboratories, Inc., Hercules, CA, USA).
Statistical analysis. All data were expressed as mean \pm SEM and analyzed by the Graphpad Prism 7.0 software using one-way analysis of variance (ANOVA) followed by Tukey's test. $\mathrm{P}<0.05$ was considered to indicate a statistically significant difference.

\section{Results}

ISO-1 reduced the serum pancreatic enzymes and pancreatic histology. Since elevated activities of serum AMY and lipase (LIPA) are considered the most sensitive and specific markers of AP (28), firstly, we assessed the activities of these markers. As shown in Fig. 1A-B, compared with SO group, serum AMY and LIPA levels were dramatically increased in the rats of APIP group $(\mathrm{P}<0.01)$. However, ISO-1 pretreatment reversed the increases compared with the APIP group $(\mathrm{P}<0.01)$.

Then pancreatic injury was estimated, based on edema, inflammatory cell infiltration, hemorrhage, and necrosis. As demonstrated in Fig. 2A, there was a little morphological evidence of pancreatic injury in SO group. While, conspicuous pancreatic edema, interstitial leukocyte infiltration, intrapancreatic hemorrhage, and necrosis were observed in the APIP group (Fig. 2B). Compared with APIP group, the extent and severity of the pancreatic histological injury were significantly alleviated in the ISO-1 group (Fig. 2C). As shown in Fig. 2D, there was a significant reduction of the pancreatic histological score in rats pretreated with ISO-1 in comparison with APIP group $(\mathrm{P}<0.01)$.

ISO-1 decreased the proinflammatory cytokines following APIP. Serum concentrations of proinflammatory cytokines such as TNF- $\alpha$, IL-1 $\beta$, and IL- 6 greatly increase in AP $(29,30)$, so we measured their levels to obtain insight into the effect of MIF antagonist ISO-1 on the inflammatory process in APIP. As illustrated in Fig. 3A-C, concomitant with the taurocholate administration, marked increases in serum levels of TNF- $\alpha$, IL-1 $\beta$ and IL-6 formation were observed in the APIP rats compared with that in SO group $(\mathrm{P}<0.01)$. By contrast, the increases of these cytokines were obviously decreased by the pharmacological blockade of MIF antagonist ISO-1 ( $\mathrm{P}<0.01)$.

ISO-1 alleviated the lung histopathology and the inflammatory cell infiltration. Next, we investigated the effect of ISO-1 on the lung injury. As shown in Fig. 4A, the lung sections of SO rats showed a normal alveolar morphology. However, rats that underwent pancreatitis demonstrated the recognized features of lung injury including alveolar wall thickening, and increased exudates as well as inflammatory cell infiltration in the alveolar spaces (Fig. 4B). The intervention of MIF antagonist ISO-1 significantly amended the APIP-induced histopathologic changes of the lung (Fig. 4C). In addition, the histopathological score of lung injury in the ISO-1 group was significantly reduced than the score of the APIP group ( $\mathrm{P}<0.01$; Fig. 4D).

Acute lung injury is usually accompanied by increased inflammatory cells accumulation, mainly including neutrophil, which can be marked by MPO (31). So, we explored whether ISO-1 could alleviate the inflammatory cell infiltration in the lung by performing immunofluorescent assay of MPO. As shown in Fig. 5A, green-colored MPO-positive cells in the lung were indicative of neutrophil cells. The number of MPO-positive cells was significantly elevated after 

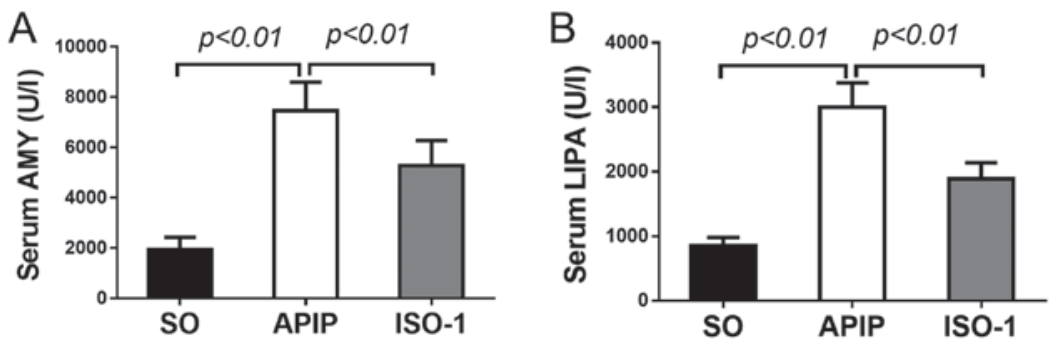

Figure 1. Effects of ISO-1 on serum AMY and LIPA in all group of rats. Serum AMY (A) and LIPA (B) levels of each group were detected by the automatic biochemical analyzer and duplicated three times. $\mathrm{P}<0.05$ indicates a significant difference between the marked groups. SO, sham operation group; APIP acute pancreatitis in pregnancy group; ISO-1, ISO-1+APIP group. ISO-1, (S,R)3-(4-hydroxyphenyl)-4,5-dihydro-5-isoxazole acetic acid methyl ester; AMY, amylase; LIPA, lipase.
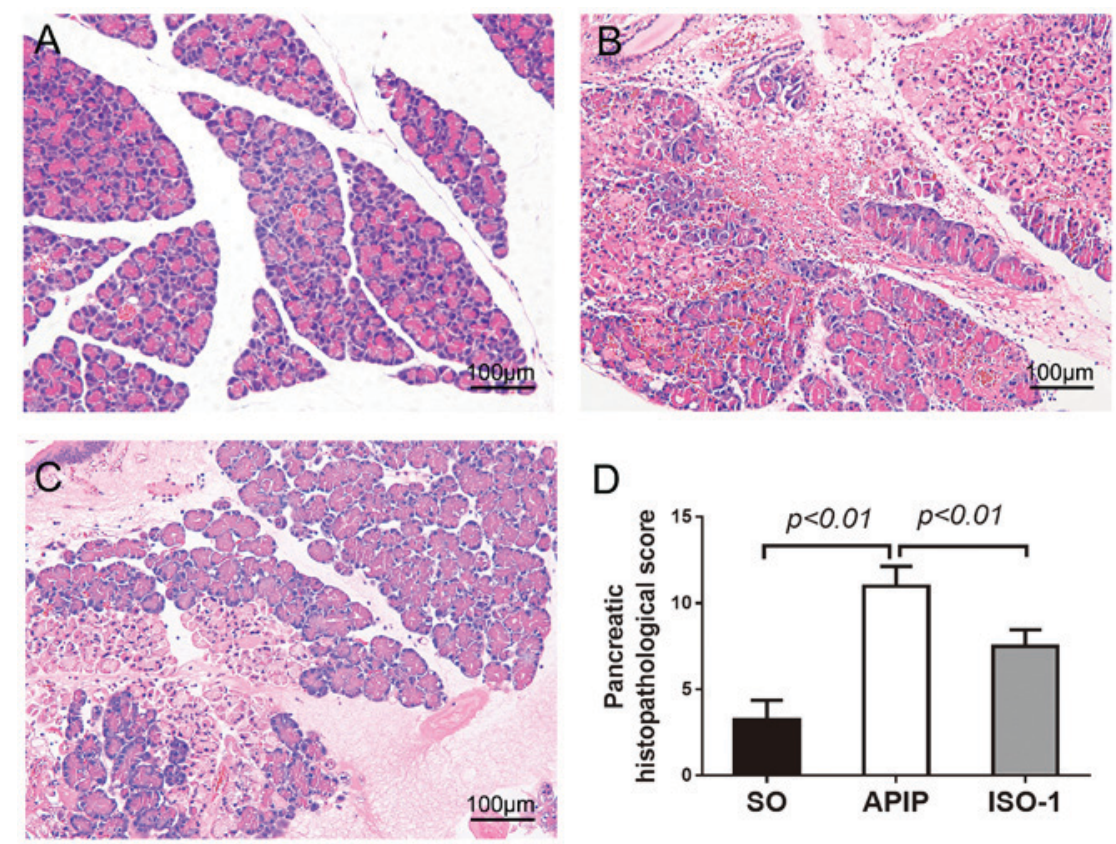

Figure 2. Morphologic changes and histopathological score of pancreas in all groups. H\&E sections were examined by light microscopy (original magnification, x200). (A) SO group, (B) APIP group, (C) ISO-1 group, (D) Comparison of the total pathological scores of pancreas in all groups. P $<0.05$ indicates a significant difference between the marked groups. SO, sham operation group; APIP, acute pancreatitis in pregnancy group; ISO-1, ISO-1+APIP group. ISO-1, (S,R)3-(4-hydroxyphenyl)-4,5-dihydro-5-isoxazole acetic acid methyl ester.
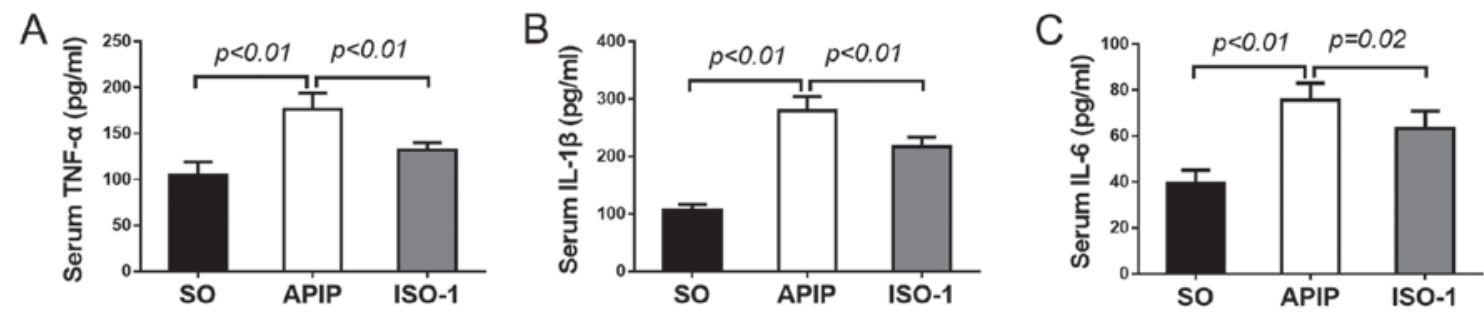

Figure 3. Effects of ISO-1 on the activities of serum proinflammatory cytokines in all groups. (A) TNF- $\alpha$, (B) IL- $1 \beta$, and (C) IL- 6 concentrations were quantified by ELISA assay. (mean $\pm \mathrm{SD}, \mathrm{n}=6$ ). $\mathrm{P}<0.05$ indicates a significant difference between the marked groups. SO, sham operation group; APIP, acute pancreatitis in pregnancy group; ISO-1, ISO-1+APIP group. ISO-1, (S,R)3-(4-hydroxyphenyl)-4,5-dihydro-5-isoxazole acetic acid methyl ester; TNF- $\alpha$, tumor necrosis factor- $\alpha$; IL, interleukin.

taurocholate induction compared to the $\mathrm{SO}$ group $(\mathrm{P}<0.01)$. In contrast, when pretreated with ISO-1, the number was apparently reduced $(\mathrm{P}<0.01 ;$ Fig. 5B).

MIF was activated in the lung of APIP rats. Abnormal MIF expression is regarded as an important process in inflammatory reaction $(32,33)$. Here, results from western blot suggested that compared with the SO group, the expression of MIF in lung tissues of APIP rats was significantly increased $(\mathrm{P}<0.01)$. ISO-1 pretreatment slightly decreased the MIF expression, but there was no statistical difference ( $\mathrm{P}>0.05$; Fig. 6A). 

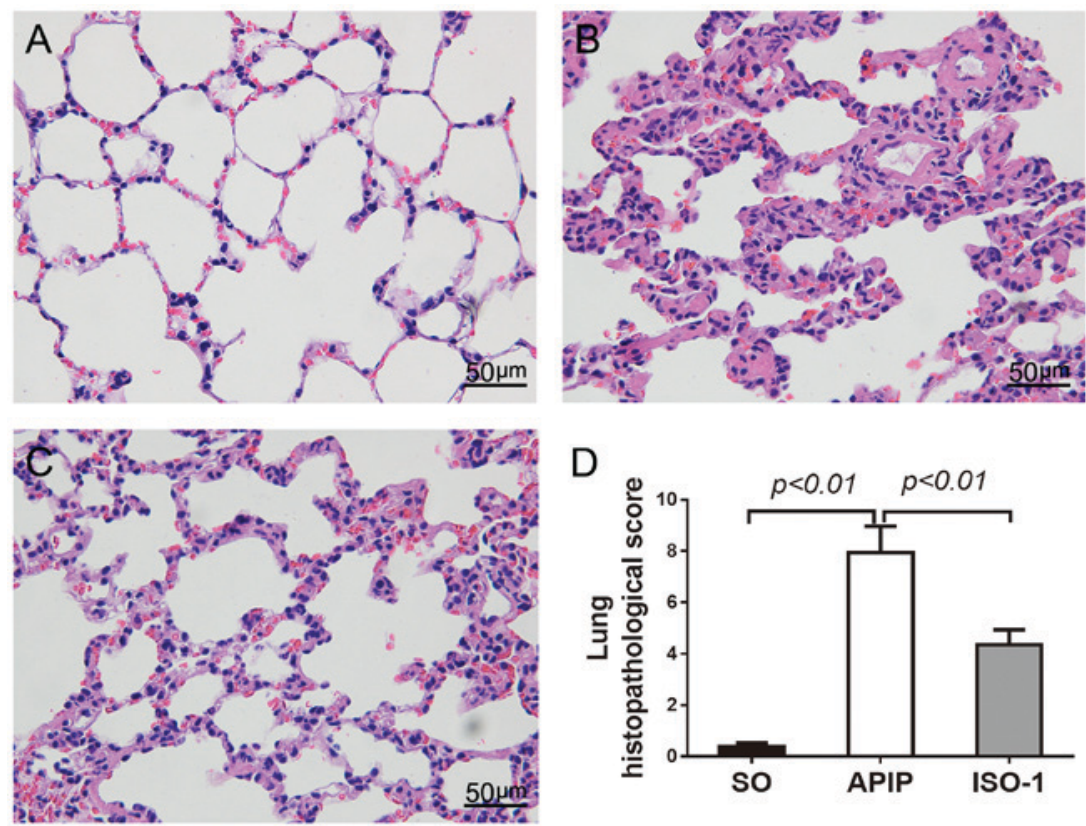

Figure 4. Representative histopathological changes of lung injury in each group (original magnification, x400). (A) SO group, (B) APIP group, (C) ISO-1 group, (D) Comparison of the total pathological scores of lung injury. $\mathrm{P}<0.05$ indicates a significant difference between the marked groups. SO, sham operation group; APIP, acute pancreatitis in pregnancy group; ISO-1, ISO-1+APIP group. ISO-1, (S,R)3-(4-hydroxyphenyl)-4,5-dihydro-5-isoxazole acetic acid methyl ester.

A

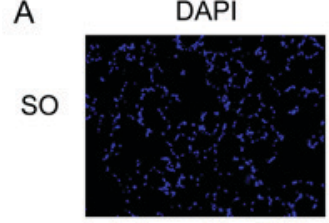

APIP

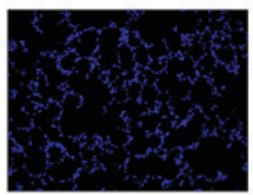

ISO-1

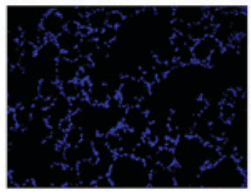

B

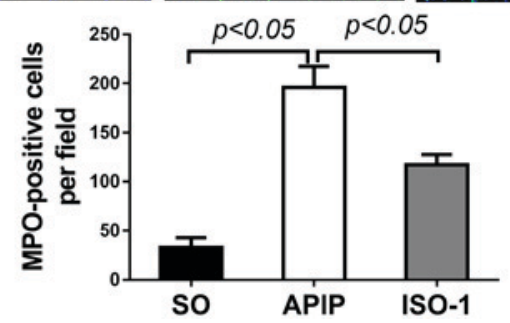

Figure 5. Representative immunofluorescence staining for MPO of the lung sections of each group (original magnification, x200). (A) MPO was stained green and nuclei was stained blue. (B) Comparison of the number of MPO-positive cells in the lung. $\mathrm{P}<0.05$ indicates a significant difference between the marked groups. SO, sham operation group; APIP, acute pancreatitis in pregnancy group; ISO-1, ISO-1+APIP group. ISO-1, (S,R)3-(4-hydroxyphenyl)-4,5-dihydro-5-isoxazole acetic acid methyl ester; $\mathrm{MPO}$, myeloperoxidase.

ISO-1 inhibited the P38MAPK and NF- $\kappa B$ activation in the lung. Taken together, all of the above results indicated that MIF antagonist ISO-1 mitigated the degree of pancreatitis and associated lung injury, but the underlying mechanism

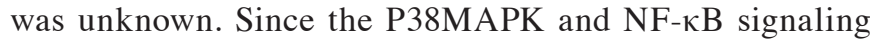
pathways play key roles in the induction of several inflammatory diseases $(34,35)$, next we investigated if ISO-1 exerts its anti-inflammatory activities by affecting the two pathways in lung injury induced by APIP. As shown in Fig. 6B, APIP induced a marked increase in the phosphorylated level of P38MAPK compared with SO group $(\mathrm{P}<0.01)$. In addition, pretreated with the MIF antagonist ISO-1 markedly reduced the phosphorylation of P38MAPK in the lung following APIP.

The translocation of $N F-\kappa B$ indicated the activation of $\mathrm{NF}-\kappa \mathrm{B}$ signaling pathway. As the western blot showed in Fig. $6 \mathrm{C}$, the expression of $\mathrm{NF}-\kappa \mathrm{B}$ increased significantly in the nucleus following APIP, accompanied by the reduction in the cytoplasm. However, pretreatment with ISO-1 inhibited this translocation process. We also found that the expression of TNF- $\alpha$ in lung tissues was greatly upregulated after APIP, but ISO-1 treatment reversed the increase $(\mathrm{P}<0.01$; Fig. 6D).

\section{Discussion}

In the present study, the effect of MIF antagonist ISO-1 on APIP and associated lung injury as well as the underlying mechanism was preliminarily explored. Our results indicated that MIF expression was upregulated in the lung of APIP rats, and ISO-1 pretreatment could ameliorate pancreatitis and associated lung injury. In addition, ISO-1 reduced serum AMY and LIPA concentrations, pro-inflammatory mediators and inflammatory cell infiltration in the lung. We also demonstrated that the inhibitory effect of ISO-1 on APIP and associated lung injury may be through deactivating P38MAPK and NF- $\kappa$ B signaling pathways. All of these observations indicate that MIF antagonist ISO-1 exerts potent anti-inflammatory effects and ameliorates the degree of APIP and associated lung injury in rats. 


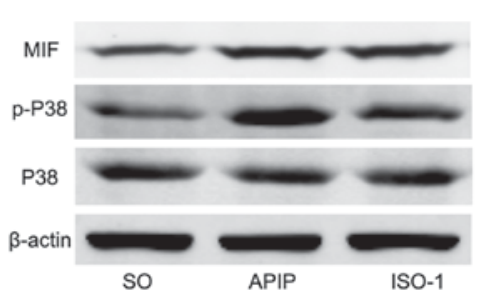

A
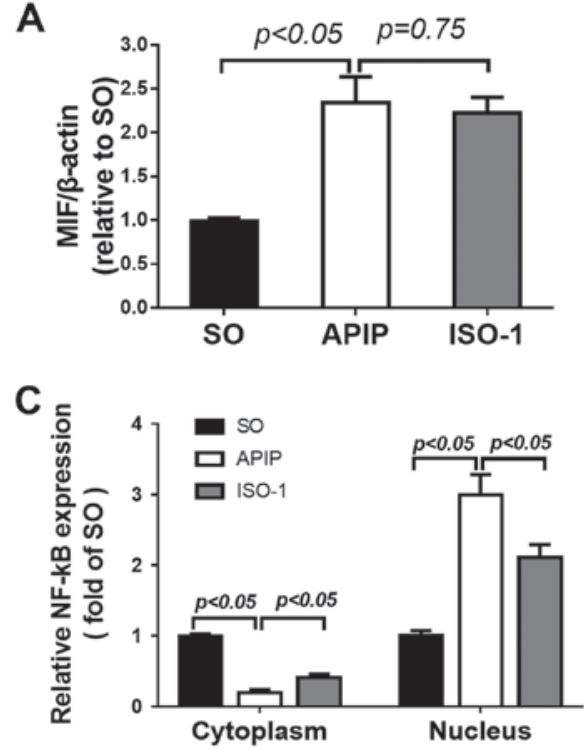

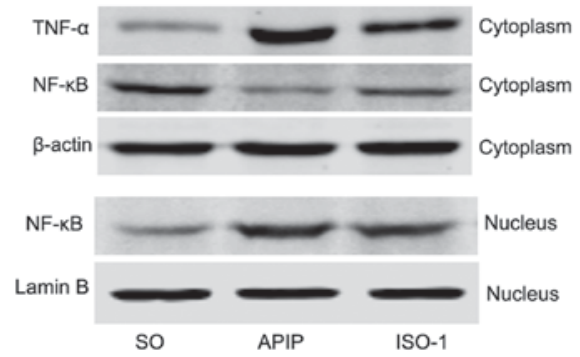

B

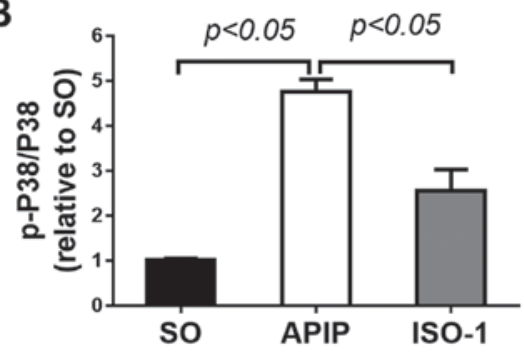

D

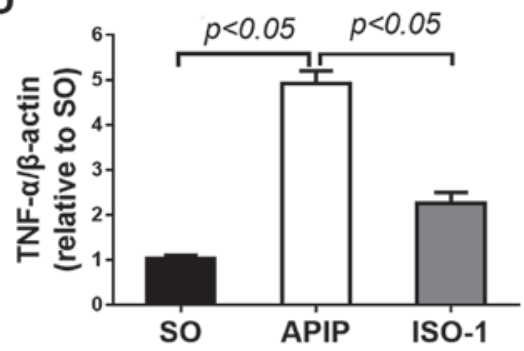

Figure 6. Effects of MIF antagonist ISO-1 on the P38MAPK and NF-кB signaling pathway in the lung injury induced by APIP. Lung samples were obtained at $6 \mathrm{~h}$ after modeling. MIF, total and phosphorylated P38 and TNF- $\alpha$ in the cytoplasm, as well as NF- $\mathrm{KB}$ in the cytoplasm and nucleus were measured by western blot assay. $\beta$-actin was used as internal control of cytoplasm, and LaminB was used as nucleus internal control. Densitometry quantification of (A) MIF, (B) $\mathrm{p}-\mathrm{P} 38$, (C) NF- $\mathrm{kB}$, and (D) TNF- $\alpha$ was evaluated by the Quantity One software. Data are expressed as mean $\pm \mathrm{SD}(\mathrm{n}=6)$. P $<0.05$ indicates a significant difference between the marked groups. SO, sham operation group; APIP, acute pancreatitis in pregnancy group; ISO-1, ISO-1+APIP group. MIF, macrophage migration inhibitory factor; ISO-1, (S,R)3-(4-hydroxyphenyl)-4,5-dihydro-5-isoxazole acetic acid methyl ester; TNF- $\alpha$, tumor necrosis factor- $\alpha$; NF- $\kappa \mathrm{B}$, nuclear factor- $\kappa \mathrm{B}$

AP is a challenging clinical problem characterized by increased mortality depending on the severity. Although there have been few clinical trials with pharmacological agents, no effective treatment exists (36). APIP is a severe complication of pregnancy, which easily results in miscarriage, stillbirth and premature birth, and the fetal mortality can be up to $10-20 \%$, even $30 \%$ as reported $(37,38)$. The incidence of APIP increased during recent years with the change of dietary habits. It mostly occurs in the third trimester. The most frequent cause of APIP is gallstones, which is responsible for more than $60 \%$ of cases $(1,39)$. Gallstones are more likely to form during pregnancy because of elevated progesterone level and it induces biliary hypotonia and increase the pressure of Oddi sphincter, which would lead to bile stasis and stone formation (40). So retrograde infusion of sodium taurocholate solution into the biliary-pancreatic duct to induce APIP is in line with the pathophysiological changes of APIP.

MIF, as an important multifunctional cytokine, involves in various physiological and pathological processes including inflammation, immunity, tumor, and pregnancy. Studies have shown that MIF plays a certain role in the initiation and development of acute necrotizing pancreatitis (41), liver injury (42), acute respiratory distress syndrome (43) and sepsis (18). ISO-1 can selectively bind to the MIF tautomerase site, inhibiting its enzyme activity, thereby suppressing some of the biological function of MIF. Lung injury is the most common organ damage in AP and is responsible for $43 \%$ mortality, a main reason for the death of AP patients (5). However, whether inhibition of MIF with ISO-1 could ameliorate lung injury induced by APIP has been unknown. Therefore, we hypothesized that MIF may be related to acute lung injury induced by APIP. In our study, it was demonstrated that MIF was significantly upregulated in the lung of APIP rats, suggesting it may be involved in the pathogenesis of acute lung injury. While, ISO-1 pretreated did not downregulate the MIF expression, which was consistent with the earlier study that ISO-1 can 'bind-onto' the tautomerase site of MIF thereby blocking its recognition whereas it can't inhibit MIF synthesis (17).

Generally, pancreatic digestive enzymes such as AMY and LIPA are most commonly obtained as the biochemical marker of pancreatic disease, particularly AP. It is contributed at an early stage to the damage of acinar cells and, consequently, to inflammatory processes and cytokine production into the pancreas. In our experiments, conspicuous hyperamylasemia, hyperlipasemia, and pathological evidences like pancreatic hemorrhage and necrosis were observed in the sodium taurocholate induced APIP rats. These results showed that the APIP model was successfully induced. 
In AP, inflammatory response and pro-inflammatory cytokines play pivotal roles and exert major influences on the outcome of the disease, in particular by triggering the systemic inflammatory response and multisystem organ failure $(44,45)$. Growing evidences have identified crucial contribution of inflammation to AP-induced lung injury. In the study, elevated levels of TNF- $\alpha$, IL-1 $\beta$, and IL- 6 in the serum of APIP rats were observed. The proinflammatory cytokine TNF- $\alpha$ is regarded as one of the key cytokine initiators of the inflammatory cascade of AP and the degree of pancreatic injury in AP correlates directly with the level of TNF- $\alpha$ (46). IL-1 $\beta$ and IL- 6 are the principal mediators in the synthesis of acute-phase proteins and in the regulation of immune responses and the inflammatory process (47). In acute lung injury, these inflammatory mediators have cytotoxic effects, such as inducing apoptosis of multiple cells including alveolar epithelial cells, increasing capillary permeability and damaging intercellular tight junctions, further resulting in increasing extravasation of vascular fluid, inflammatory cells and more inflammatory mediators (48). In our study, recognized features of lung injury including alveolar wall thickening, and increased exudates as well as inflammatory cell infiltration in the alveolar spaces of rats that underwent pancreatitis were observed.

MPO has been used as a biochemical marker for inflammatory cells infiltration in studies of multiple-organ injury in AP (49). In addition, its activity in the lung were correlated with the degree of lung injury (31). In this study, the number of MPO-positive cells were dramatically increased in the lung of APIP rats. Together with aggravating morphological changes of the lung and increased inflammatory cells infiltration demonstrated obvious lung injury during the progression of pancreatitis.

However, ISO-1 pretreatment greatly inhibited the elevation of serum AMY and LIPA, in addition, we also found that ISO-1 significantly improved the pathological state of the pancreas and lung, inhibited proinflammatory cytokines, and reduced the number of MPO-positive cells. All of these observations indicate that the MIF antagonist ISO-1 exerts potent anti-inflammatory effects and ameliorates the degree of APIP and associated lung injury in rats.

Published studies have showed P38MAPK and NF- $\kappa \mathrm{B}$ are both essential pathways involved in regulating the expression of inflammatory mediators in the pathogenesis of SAP $(50,51)$. The expression of phosphorylated P38MAPK in the pancreatic and lung tissue was increased rapidly in the SAP rat model (50). And inhibiting P38MAPK expression ameliorates the severity of the disease (52). Therefore, P38MAPK activation may represent a major regulatory mechanism during severe acute pancreatitis. Our results revealed that taurocholate stimulation could induce the phosphorylation of P38MAPK in the lung tissue. As anticipated, ISO-1 pretreatment greatly inhibited the APIP-induced P38MAPK phosphorylation. In addition, we found that the MIF antagonist ISO-1 can simultaneously inhibit NF- $\kappa \mathrm{B}$ signaling pathway in the lung. Activation of NF- $\mathrm{KB}$ pathway needs NF- $\mathrm{kB}$ translocation from cell plasma to the nucleus, binding to the promoter region of various pro-inflammatory NF- $\mathrm{kB}$ responsive genes and activates transcription (53). Our data showed that NF- $\kappa B$ increased significantly in the nucleus following APIP-induced lung injury, accompanied by the reduction in the cytoplasm, implying the activation of NF- $\mathrm{KB}$ signaling pathway. We found that pretreatment with ISO-1 effectively attenuated NF- $\kappa \mathrm{B}$ intranuclear translocation in the lung. Inhibition of NF- $\kappa \mathrm{B}$ activation reduces the severity of severe acute pancreatitis (54). ISO-1 inhibits the NF- $\kappa \mathrm{B}$ activation and thus ameliorates severe acute pancreatitis-induced lung injury. Previous studies have shown that NF- $\kappa \mathrm{B}$ activating can produce multiple proinflammatory cytokines (55). In this study, we detected the expression of TNF- $\alpha$ in the lung tissues. The results showed that the expression of TNF- $\alpha$ was greatly increased in the lung of APIP rats, whereas ISO-1 pretreatment reduced the increase. These results indicate that the protective effects of ISO-1 against lung injury induced by APIP are correlated with the deactivation of P38MAPK and NF- $\mathrm{KB}$ signaling pathways.

In summary, our study provides evidences that MIF upregulation participated in the lung injury following APIP, and ISO-1, an MIF antagonist, markedly ameliorated the severity of pancreatitis and lung injury, which may through the deactivation of P38MAPK and NF- $\kappa \mathrm{B}$ signaling pathways. Targeting its upstream or downstream substrates may attenuate lung injury and improve APIP outcomes, but the specific mechanisms need further investigations. Therefore, the findings presented in this study may stimulate interest in the development of more potent and specific MIF inhibitors for the prevention and treatment of APIP, and associated lung injury.

\section{Acknowledgements}

The authors are grateful to the Central Laboratory, Hubei Key Laboratory of Digestive System Disease of Renmin Hospital of Wuhan University for providing relevant experimental facilities and technical support.

\section{Funding}

This study was supported by the National Natural Science Foundation of China (no. 81370562).

\section{Availability of data and materials}

The datasets used and/or analyzed during the current study are available from the corresponding author on reasonable request.

\section{Authors' contributions}

YZ designed the study, performed the majority of experiments and wrote this article. LZ and YD analyzed the data and helped to write the paper. FM and YH performed the immunofluorescence and western blot assay. HX performed the animal experiments. TZ participated in the design of the study and performed the ELISA assay. WW participated in the design of this study and provided financial support for this work. All authors read and approved the final manuscript.

\section{Ethics approval and consent to participate}

All animal experiments in this study were reviewed and approved by the Ethics Committee of Wuhan University and performed in compliance with the ARRIVE guidelines. 


\section{Consent for publication}

Not applicable.

\section{Competing interests}

All the authors declare that they have no competing interests.

\section{References}

1. Mali P: Pancreatitis in pregnancy: Etiology, diagnosis, treatment, and outcomes. Hepatobiliary Pancreat Dis Int 15: 434-438, 2016

2. Zuo T, Yu J, Wang WX, Zhao KL, Chen C, Deng WH, He XB, Wang P, Shi Q and Guo WY: Mitogen-activated protein kinases are activated in placental injury in rat model of acute pancreatitis in pregnancy. Pancreas 45: 850-857, 2016.

3. Akcakaya A, Ozkan OV, Okan I, Kocaman O and Sahin M: Endoscopic retrograde cholangiopancreatography during pregnancy without radiation. World J Gastroenterol 15: 3649-3652, 2009.

4. Hernandez A, Petrov MS, Brooks DC, Banks PA, Ashley SW and Tavakkolizadeh A: Acute pancreatitis and pregnancy: A 10-year single center experience. J Gastrointest Surg 11: 1623-1627, 2007.

5. Halonen KI, Pettilä V, Leppäniemi AK, Kemppainen EA, Puolakkainen PA and Haapiainen RK: Multiple organ dysfunction associated with severe acute pancreatitis. Crit Care Med 30 1274-1279, 2002.

6. Mole DJ, Webster SP, Uings I, Zheng X, Binnie M, Wilson K, Hutchinson JP, Mirguet O, Walker A, Beaufils B, et al: Kynurenine-3-monooxygenase inhibition prevents multiple organ failure in rodent models of acute pancreatitis. Nat Med 22: 202-209, 2016.

7. Elder AS, Saccone GT and Dixon DL: Lung injury in acute pancreatitis: Mechanisms underlying augmented secondary injury. Pancreatology 12: 49-56, 2012.

8. Pastor CM, Matthay MA and Frossard JL: Pancreatitis-associated acute lung injury: New insights. Chest 124: 2341-2351, 2003.

9. Jaffray C, Yang J, Carter G, Mendez C and Norman J: Pancreatic elastase activates pulmonary nuclear factor kappa B and inhibitory kappa $\mathrm{B}$, mimicking pancreatitis-associated adult respiratory distress syndrome. Surgery 128: 225-231, 2000.

10. Lu H, Bai Y, Wu L, Hong W, Liang Y, Chen B and Bai Y: Inhibition of macrophage migration inhibitory factor protects against inflammation and matrix deposition in kidney tissues after injury. Mediat Inflamm 2016: 2174682, 2016.

11. Leng L, Metz CN, Fang Y, Xu J, Donnelly S, Baugh J, Delohery T, Chen Y, Mitchell RA and Bucala R: MIF signal transduction initiated by binding to CD74. J Exp Med 197: 1467-1476, 2003

12. Lue H, Thiele M, Franz J, Dahl E, Speckgens S, Leng L, Fingerle-Rowson G, Bucala R, Lüscher B and Bernhagen J: Macrophage migration inhibitory factor (MIF) promotes cell survival by activation of the Akt pathway and role for CSN5/JAB1 in the control of autocrine MIF activity. Oncogene 26: 5046-5059, 2007.

13. Gao Y, Hou R, Liu F, Liu H, Fei Q, Han Y, Cai R, Peng C and Qi Y: Obacunone causes sustained expression of MKP-1 thus inactivating p38 MAPK to suppress pro-inflammatory mediators through intracellular MIF. J Cell Biochem 119: 837-849, 2018.

14. Roger T, Schneider A, Weier M, Sweep FC, Le Roy D, Bernhagen J, Calandra T and Giannoni E: High expression levels of macrophage migration inhibitory factor sustain the innate immune responses of neonates. Proc Natl Acad Sci USA 113: E997-E1005, 2016.

15. Aeberli D, Leech M and Morand EF: Macrophage migration inhibitory factor and glucocorticoid sensitivity. Rheumatology (Oxford) 45: 937-943, 2006.

16. Calandra T, Echtenacher B, Roy DL, Pugin J, Metz CN, Hültner L, Heumann D, Männel D, Bucala R and Glauser MP: Protection from septic shock by neutralization of macrophage migration inhibitory factor. Nat Med 6: 164-170, 2000.

17. Al-Abed Y, Dabideen D, Aljabari B, Valster A, Messmer D, Ochani M, Tanovic M, Ochani K, Bacher M, Nicoletti F, et al: ISO-1 binding to the tautomerase active site of MIF inhibits its pro-inflammatory activity and increases survival in severe sepsis. J Biol Chem 280: 36541-36544, 2005.
18. Bozza FA, Gomes RN, Japiassú AM, Soares M, Castro-Faria-Neto HC, Bozza PT and Bozza MT: Macrophage migration inhibitory factor levels correlate with fatal outcome in sepsis. Shock 22: 309-313, 2004.

19. Mikulowska A, Metz CN, Bucala R and Holmdahl R: Macrophage migration inhibitory factor is involved in the pathogenesis of collagen type II-induced arthritis in mice. J Immunol 158: 5514-5517, 1997.

20. Amano T, Nishihira J and Miki I: Blockade of macrophage migration inhibitory factor (MIF) prevents the antigen-induced response in a murine model of allergic airway inflammation. Inflamm Res 56: 24-31. 2007.

21. Ohkawara T, Nishihira J, Takeda H, Hige S, Kato M, Sugiyama T, Iwanaga T, Nakamura $\mathrm{H}$, Mizue Y and Asaka M: Amelioration of dextran sulfate sodium-induced colitis by anti-macrophage migration inhibitory factor antibody in mice. Gastroenterology 123: 256-270, 2002.

22. Russell KE, Chung KF, Clarke CJ, Durham AL, Mallia P, Footitt J, Johnston SL, Barnes PJ, Hall SR, Simpson KD, et al: The MIF antagonist ISO-1 attenuates corticosteroid-insensitive inflammation and airways hyperresponsiveness in an ozone-induced model of COPD. PLoS One 11: e146102, 2016.

23. West PW, Parker LC, Ward JR and Sabroe I: Differential and cell-type specific regulation of responses to Toll-like receptor agonists by ISO-1. Immunology 125: 101-110, 2008.

24. Yu J, Deng W, Wang W, Ding Y, Jin H, Chen C, Chen X, Xiong X and Xu S: Inhibition of poly(ADP-ribose) polymerase attenuates acute kidney injury in sodium taurocholate-induced acute pancreatitis in rats. Pancreas 41: 1299-1305, 2012.

25. Mei F, Shi Q, Zuo T, Chen C, Wang P, Li C, He B, Yang X, Hu P and Wang W: Dose-effect relationship and protective effect of MIF inhibitor on pancreas and placenta injuries in rats with acute necrotizing pancreatitis in late pregnancy. Chin J Emerg Med 25: 45-49, 2016

26. Schmidt J, Rattner DW, Lewandrowski K, Compton CC, Mandavilli U, Knoefel WT and Warshaw AL: A better model of acute pancreatitis for evaluating therapy. Ann Surg 215: 44-56, 1992.

27. Werner J, Z'Graggen K, Fernández-del Castillo C, Lewandrowski KB, Compton CC and Warshaw AL: Specific therapy for local and systemic complications of acute pancreatitis with monoclonal antibodies against ICAM-1. Ann Surg 229: 834-842, 1999.

28. Sha H, Ma Q, Jha RK and Wang Z: Resveratrol ameliorates lung injury via inhibition of apoptosis in rats with severe acute pancreatitis. Exp Lung Res 35: 344-358, 2009.

29. Nishiki Y, Adewola A, Hatanaka M, Templin AT, Maier B and Mirmira RG: Translational control of inducible nitric oxide synthase by p38 MAPK in islet $\beta$-cells. Mol Endocrinol 27: 336-349, 2013

30. Que RS, Cao LP, Ding GP, Hu JA, Mao KJ and Wang GF: Correlation of nitric oxide and other free radicals with the severity of acute pancreatitis and complicated systemic inflammatory response syndrome. Pancreas 39: 536-540, 2010.

31. Kim K, Li Y, Jin G, Chong W, Liu B, Lu J, Lee K, Demoya M, Velmahos GC and Alam HB: Effect of valproic acid on acute lung injury in a rodent model of intestinal ischemia reperfusion. Resuscitation 83: 243-248, 2012.

32. Bargagli E, Olivieri C, Nikiforakis N, Cintorino M, Magi B, Perari MG, Vagaggini C, Spina D, Prasse A and Rottoli P: Analysis of macrophage migration inhibitory factor (MIF) in patients with idiopathic pulmonary fibrosis. Respir Physiol Neurobiol 167: 261-267, 2009.

33. Heinrichs D, Knauel M, Offermanns C, Berres ML, Nellen A, Leng L, Schmitz P, Bucala R, Trautwein C, Weber C, et al: Macrophage migration inhibitory factor (MIF) exerts antifibrotic effects in experimental liver fibrosis via CD74. Proc Natl Acad Sci USA 108: 17444-17449, 2011.

34. Jaworek J, Jachimczak B, Tomaszewska R, Konturek PC, Pawlik WW, Sendur R, Hahn EG, Stachura J and Konturek SJ: Protective action of lipopolysaccharidesin rat caerulein-induced pancreatitis: Role of nitric oxide. Digestion 62: 1-13, 2000.

35. Gao Y, Liu F, Fang L, Cai R, Zong C and Qi Y: Genkwanin inhibits proinflammatory mediators mainly through the regulation of miR-101/MKP-1/MAPK pathway in LPS-activated macrophages. PLoS One 9: e96741, 2014.

36. Choi SB, Bae GS, Jo IJ, Wang S, Song HJ and Park SJ: Berberine inhibits inflammatory mediators and attenuates acute pancreatitis through deactivation of JNK signaling pathways. Mol Immunol 74: 27-38, 2016. 
37. Geng Y, Li W, Sun L, Tong Z, Li N and Li J: Severe acute pancreatitis during pregnancy: Eleven years experience from a surgical intensive care unit. Dig Dis Sci 56: 3672-3677, 2011.

38. Stimac D and Stimac T: Acute pancreatitis during pregnancy. Eur J Gastroenterol Hepatol 23: 839-844, 2011.

39. Hacker FM, Whalen PS, Lee VR and Caughey AB: Maternal and fetal outcomes of pancreatitis in pregnancy. Am J Obstet Gynecol 213: 568.e1-5, 2015 .

40. Robertson KW, Stewart IS and Imrie CW: Severe acute pancreatitis and pregnancy. Pancreatology 6: 309-315, 2006.

41. Rahman SH, Menon KV, Holmfield JH, McMahon MJ and Guillou JP: Serum macrophage migration inhibitory factor is an early marker of pancreatic necrosis in acute pancreatitis. Ann Surg 245: 282-289, 2007.

42. Barnes MA, McMullen MR, Roychowdhury S, Pisano SG, Liu X, Stavitsky AB, Bucala R and Nagy LE: Macrophage migration inhibitory factor contributes to ethanol-induced liver injury by mediating cell injury, steatohepatitis, and steatosis. Hepatology 57: 1980-1991, 2013

43. Lai KN, Leung JC, Metz CN, Lai FM, Bucala R and Lan HY: Role for macrophage migration inhibitory factor in acute respiratory distress syndrome. J Pathol 199: 496-508, 2003.

44. Norman J, Franz M, Messina J, Riker A, Fabri PJ, Rosemurgy AS and Gower WR Jr: Interleukin-1 receptor antagonist decrease severity of experimental acute pancreatitis. Surgery 117: 648-655, 1995.

45. Hughes CB, Grewal HP, Gaber LW, Kotb M, El-din AB, Mann L and Gaber AO: Anti-TNFalpha therapy improves survival and ameliorates the pathophysiologic sequelae in acute pancreatitis in the rat. Am J Surg 171: 274-280, 1996.

46. Baker SJ and Reddy EP: Transducers of life and death: TNF receptor superfamily and associated proteins. Oncogene 12: 1-9, 1996.

47. Papachristou GI: Prediction of severe acute pancreatitis: Current knowledge and novel insights. World J Gastroenterol 14: 6273-6275, 2008.
48. Lukacs NW and Ward PA: Inflammatory mediators, cytokines, and adhesion molecules in pulmonary inflammation and injury. Adv Immunol 62: 257-304, 1996.

49. Chen C, Xu S, Wang WX, Ding YM, Yu KH, Wang B and Chen XY: Rosiglitazone attenuates the severity of sodium taurocholate-induced acute pancreatitis and pancreatitis-associated lung injury. Arch Med Res 40: 79-88, 2009.

50. Twait E, Williard DE and Samuel I: Dominant negative p38 mitogen-activated protein kinase expression inhibits NF-kappaB activation in AR42J cells. Pancreatology 10: 119-128, 2010.

51. Lv W, Lv C, Yu S, Yang Y, Kong H, Xie J, Sun H, Andersson R, $\mathrm{Xu}$ D, Chen B and Zhou M: Lipoxin A4 attenuation of endothelial inflammation response mimicking pancreatitis-induced lung injury. Exp Biol Med (Maywood) 238: 1388-1395. 2013.

52. Chen P, Zhang Y, Qiao M and Yuan Y: Activated protein C, an anticoagulant polypeptide, ameliorates severe acute pancreatitis via regulation of mitogen-activated protein kinases. J Gastroenterol 42: 887-896, 2007.

53. Zhong H, SuYang H, Erdjument-Bromage H, Tempst $\mathrm{P}$ and Ghosh S: The transcriptional activity of NF-kappaB is regulated by the IkappaB-associated PKAc subunit through a cyclic AMP-independent mechanism. Cell 89: 413-424, 1997.

54. Yubero S, Ramudo L, Manso MA and De Dios I: Mechanisms of dexamethasone-mediated chemokine down-regulation in mild and severe acute pancreatitis. Biochim Biophys Acta 1792: 1205-1211, 2009.

55. Kim C, Sano Y, Todorova K, Carlson BA, Arpa L, Celada A, Lawrence T, Otsu K, Brissette JL, Arthur JS and Park JM: The kinase p38 alpha serves cell type-specific inflammatory functions in skin injury and coordinates pro- and anti-inflammatory gene expression. Nat Immunol 9: 1019-1027, 2008.

This work is licensed under a Creative Commons Attribution-NonCommercial-NoDerivatives 4.0 International (CC BY-NC-ND 4.0) License. 